\title{
Erratum to: Comparison of pulpal anesthesia and cardiovascular parameters with lidocaine with epinephrine and lidocaine with clonidine after maxillary infiltration in type 2 diabetic volunteers
}

\author{
Marija S. Milic ${ }^{1}$ - Bozidar Brkovic ${ }^{1}$ Elena Krsljak ${ }^{2}$ - Dragica Stojic ${ }^{3}$
}

Published online: 22 October 2015

(C) Springer-Verlag Berlin Heidelberg 2015

Erratum to: Clinical Oral Investigations

DOI 10.1007/s00784-015-1610-5

The original version of the above article contained a mistake in the arrangement of Table 2. Below is the correct table.

The online version of the original article can be found at http://dx.doi.org/ 10.1007/s00784-015-1610-5.

\footnotetext{
Dragica Stojic

dragica.stojic@stomf.bg.ac.rs

1 Clinic of Oral Surgery, School of Dental Medicine, University of Belgrade, Belgrade, Serbia

2 Department of Physiology, School of Dental Medicine, University of Belgrade, Belgrade, Serbia

3 Department of Pharmacology in Dentistry, School of Dental Medicine, University of Belgrade, Belgrade, Serbia
} 
Table 2 Pulpal blood flow

\begin{tabular}{|c|c|c|c|c|c|c|c|c|}
\hline \multirow[t]{3}{*}{ Observation time (min) } & \multicolumn{5}{|l|}{ Participants } & \multirow{2}{*}{\multicolumn{3}{|c|}{$\mathrm{p}^{\mathrm{a}}$}} \\
\hline & \multicolumn{2}{|c|}{ Healthy $(n=36)$} & \multicolumn{3}{|c|}{ Diabetic $(n=36)$} & & & \\
\hline & H-LC & H-LE & DM-LC & DM-LE & $\mathrm{p}^{\mathrm{b}}$ & Group difference & Time course & Interaction \\
\hline 0 (baseline) & $4.74 \pm 1.45$ & $4.40 \pm 1.33$ & $4.99 \pm 1.61$ & $5.19 \pm 1.66$ & 0.094 & 0.037 & $<0.001$ & $<0.001$ \\
\hline 5 & $3.52 \pm 1.60^{*}$ & $2.87 \pm 1.16^{* \#}$ & $4.26 \pm 1.41^{*}$ & $4.40 \pm 1.63^{*}$ & 0.001 & & & \\
\hline 10 & $3.19 \pm 1.57^{*}$ & $2.45 \pm 1.05 *^{*}$ & $3.82 \pm 1.32 *$ & $3.94 \pm 1.72 *$ & 0.001 & & & \\
\hline 15 & $2.90 \pm 1.23^{*}$ & $2.26 \pm 0.98^{* \#}$ & $3.30 \pm 1.36^{*}$ & $3.55 \pm 1.52 *$ & 0.001 & & & \\
\hline 30 & $3.29 \pm 1.34 *$ & $2.89 \pm 1.13^{*}$ & $3.75 \pm 1.53^{*}$ & $3.27 \pm 1.37 *$ & 0.065 & & & \\
\hline 45 & $3.80 \pm 1.12 *$ & $3.66 \pm 1.25^{*}$ & $4.29 \pm 1.59^{* \#}$ & $3.34 \pm 1.30^{*}$ & 0.025 & & & \\
\hline 60 & $4.57 \pm 1.37$ & $4.31 \pm 1.37$ & $4.79 \pm 1.83^{\#}$ & $3.27 \pm 1.29 *$ & 0.005 & & & \\
\hline $\mathrm{p}^{\mathrm{c}}$ & 0.001 & $<0.001$ & 0.005 & $<0.001$ & & & & \\
\hline
\end{tabular}

Values given as mean $\pm \mathrm{SD}$ in perfusion units (PU)

$n$ number of participants, $H$ Healthy participants, $D M$ Diabetes Mellitus Type 2, $L C$ Lidocaine with Clonidine, $L E$ Lidocaine with Epinephrine

${ }^{\mathrm{a}}$ Two-way repeated measures ANOVA for main effects and interaction, ${ }^{\mathrm{b}}$ one-way ANOVA, Tuckey post hoc $\left({ }^{*} p<0.05\right.$, H-LE vs. DM-LE 5 th min, 10 th min, 15th min; DM-LC vs. DM-LE 45th min, 60th min), ${ }^{\mathrm{c}}$ one-way repeated measures ANOVA, Bonferroni post hoc $\left({ }^{*} p<0.05,0\right.$ (baseline) vs. observed time interval) 